\title{
Germanica
}

\section{Neue Sachlichkeit als ethische Haltung}

La Nouvelle Objectivité: une attitude éthique

\section{Pierre Vaydat}

\section{(2) OpenEdition}

\section{Journals}

Édition électronique

URL : http://journals.openedition.org/germanica/2381

DOI : 10.4000/germanica.2381

ISSN : 2107-0784

Éditeur

Université de Lille

\section{Édition imprimée}

Date de publication : 31 décembre 1991

Pagination : 37-54

ISSN : 0984-2632

Référence électronique

Pierre Vaydat, « Neue Sachlichkeit als ethische Haltung », Germanica [Online], 9 | 1991, Online erschienen am: 07 Juli 2014, abgerufen am 06 Oktober 2020. URL : http://journals.openedition.org/ germanica/2381 ; DOI : https://doi.org/10.4000/germanica.2381

Ce document a été généré automatiquement le 6 octobre 2020.

(c) Tous droits réservés 


\title{
Neue Sachlichkeit als ethische Haltung
}

\author{
La Nouvelle Objectivité : une attitude éthique
}

Pierre Vaydat

1 Der neusachliche Autor bzw. Lyriker vollbringt schreibend oder dichtend einen Akt der Demut im Sinne der Negation des künstlerischen Selbstgefühls und Geltungsdranges. Er verzichtet auf die Poetisierung der Wirklichkeit und die damit einhergehende Überhöhung seiner selbst, indem er die in der Hochliteratur zur Norm erhobene souveräne Handhabung der ästhetischen Mittel zurückweist. Dies tut er zum einen, um sich der sogenannten «Sache», d.h. der tunlichst nackten, soziologisch erfassten Wirklichkeit aus freien Stücken zu unterwerfen ${ }^{1}$; und andererseits, um sich in den Dienst einer aufklärerischen Aufgabe $\mathrm{zu}$ stellen. Angestrebt wird trotz der Selbstbescheidung nichts Geringeres als eine sittliche Läuterung der Kunst, die programmatisch dargestellt wird als Voraussetzung des Klarsehens. Auf diese Weise soll der gängige Stimmigkeitsanspruch der realistischen Darstellung endlich zur Echtheit werden, das Ästhetische in das Ethische übergehen, statt dass, wie sonst üblich, der umgekehrte Prozess stattfindet.

2 So spricht Horst Denkler mit Fug von einem "Arbeitsethos»" , das offenbar aufzufassen ist im Sinne einer neuartigen, an der Abbildung der Wirklichkeit orientierten Rechtschaffenheit; während Ernst Bloch umgekehrt die Neue Sachlichkeit verächtlich als «Muckertum» hinstellt, da sie einen «puritanischen Zug (...) mitten im kessesten Schein» aufweise ${ }^{3}$. Als Kracauer der Neuen Sachlichkeit in den Zwanziger Jahren noch nahestand, verkündete er diese Haltung als eine des stoischen «Wartens»: er wünschte sich den Intellektuellen, der im einstweiligen «Vakuum der Glaubenslosigkeit [...] tapfer aus(zu)harren» wisse, statt «auf Grund romantischen Willensentschlusses irgendwo Unterschlupf (zu) suchen» ${ }^{4}$. «Romantische(r) Willensentschluss»: damit ist wohl gemeint ein ästhetischer im Gegensatz zu einem moralischen. Auch bei Kracauer ist die «Wirklichkeit» eine ethische Instanz - und zwar in Ermangelung einer vielleicht auf ewig ausbleibenden neuen «Sinnesoffenbarung». Hier treffen wir auch, wohl nicht zum ersten Male, aber immerhin schon ziemlich früh, die später banal gewordene 
neusachliche Metapher der «Kühle», die hier freilich einen erheiternden Eindruck macht, da der Stil des noch recht jungen Kracauer in einem bedenklichen Masse das Stigma der Nietzsche-Diktion trägt $^{5}$.

3 Bis über 1929 hinaus, und auch nicht erst seit 1924, hat es eine Haltung des Schreibenden gegeben, die Döblin schon 1913 definiert hatte, wobei er die Kennzeichnung «Sachlichkeit» und das Adjektiv «sachlich» verwendete ${ }^{6}$. Döblin ging es schon damals nicht mehr um die Verteidigung des verlöschenden Naturalismus, sondern um neue Werte, welche die Literatur sich nur aneignen konnte durch eine moralische Bekehrung, da sie ihrer angewohnten ästhetischen Beschaulichkeit Hohn sprachen. Dass man sich damit auf dem Gebiet (oder zumindest auf dem Parkett) der sittlichen Entscheidung bewegte, liess Döblin verlauten mittels seiner angestrengten Kant-Parodie: «es wird so: der bestirnte Himmel über mir und die Eisenbahnschienen untermir ${ }^{7}$. Das klingt wie eine Geschmacklosigkeit, doch freilich nur dann, wenn man die idealistische Heuchelei im wilhelminischen Diskurs überhört. Döblin verlangt vom Schriftsteller, dass er seiner bisherigen Kunstwelt entsage, dass er sich einer Askese unterziehe, die veraltete «Psychologie» und die «verheuchelte Lyrik» verabschiede. Das «Sachliche» wird aufgefahren gegen die «Bilder» («Bilder sind bequem»). Die neue Haltung, die er erfordert, bezeichnet Döblin ganz folgerichtig, wenn auch unangenehm bombastisch, als «Fanatismus der Selbstverleugnung» oder «Fanatismus der Entäusserung». Gemeint ist, dass der Autor in der "Sache» aufzugehen und seine selbstbezogene Individualität gleichsam aufzulösen habe: «(...) ich bin nicht ich, sondern die Strasse, die Laternen, dies und dies Ereignis, weiter nichts» ${ }^{8}$. In seinem berühmten Aufsatz von 1924 mit dem Titel «Der Geist des naturalistischen Zeitalters» bringt es Döblin dann fertig, den «naturalistischen Geist» vollends als eine neue, und zwar als die von jetzt an allein massgebliche Ethik zu präsentieren. Dieser Geist ist in der Sicht von Döblin keineswegs nur gleichbedeutend mit dem Naturalismus als einer literarischen Strömung, sondern er ist der wissenschaftliche und technologische Materialismus überhaupt, der bereits seit Jahrhunderten im Kampf liegt mit der «metaphysischen Periode», in welcher «der Schwerpunkt der gesamten weltlichen Existenz im Jenseits lag». Döblin will freilich das Wort «Materialismus» nicht akzeptieren und wählt statt dessen die Bezeichnung «Naturalismus». In diesem Text, der (paradoxerweise oder vielleicht eher, weil er einen eben unleugbar vorhandenen Tatbestand spiegelt) in manchem unheimlich auf Jüngers «Arbeiter» vorzuweisen scheint, feiert Döblin den «Gesellschaftstrieb» und deutet ihn ungezwungen klischeehaft «biologisch» aus: Er schafft die wechselnden grossen neuen Verbände und ihren Geist, die Völker und Kulturen. Biologisch sind das Versuche, zu Variationen zu gelangen, neue Menschentypen $\mathrm{zu}$ bilden» ${ }^{9}$. Was beim jungen Kracauer etwa gleichzeitig noch eine gewissermassen idealistisch befangene Geschichtsmetaphysik blieb (das Zeitalter des «Wartens» zwischen dem verblassten «Absoluten» und der vorläufig nur zu erträumenden «neuen Sinnesoffenbarung»), das tritt uns bei Döblin entgegen als robustes biologistischoptimistisches Glaubensbekenntnis: «Charakteristisch für die jetzige Epoche muss sein das Kleinheitsgefühl, stammend aus der Einsicht von der verlorenen zentralen Stellung in der Welt, und das der Einsicht in die Belanglosigkeit des tierisch-menschlichen Einzelwesens. Daneben steht das Freiheits- und Unabhängigkeitsgefühl, stammend aus der Gewissheit, nicht für ein Jenseits zu leben und alles von sich aus leisten zu müssen. Mit dem Freiheitsgefühl verbindet und aus ihm wächst sofort der Antrieb zu kräftigster Aktivität. Es kommt ganz und gar nicht zur Verzweiflung nach dem Schwinden der Jenseitsgläubigkeit. Es 
wird so: der bestirnte Himmel über mir und die Eisenbahnscheinen unter mir» ${ }^{10}$. Diesem Wandel gegenüber fordert Döblin eine ethische Haltung, welche die «Entseelung» in Kauf nehme, weil diese nur die notwendige negative Seite des unabdingbaren «schweren langsamen Prozesses der Umseelung» darstelle. Im Zeichen dieser «Umseelung» leistet sich Döblin den jugendlichen Streich, nach dem KantSpruch auch den bekannten Fontane-Spruch zu profanieren: «Diese Menschen sagen wirklich statt Gott Kattun. Aber Kattun ist ihnen so wenig ein Spass wie den früheren ihr Gott» ${ }^{11}$. Gemeint sind statt wie im Stechlin die Engländer die neuen Menschen des sogenannten naturalistischen Zeitalters überhaupt, die die Technik wirklich bejahen und sich mit den neuen Entwicklungen der Maschinenkultur identifizieren.

4 Döblin ist Anfang der Zwanziger Jahre dermassen entschlossen, sich mit der «technisch-naturalistischen Kraft» $\mathrm{zu}$ identifizieren, dass er sogar dem Industriekapitalismus einen zu guter Letzt uneigennützigen, weil eben auf Grund des neuen heraufziehenden Weltzeitalters legitimen «Machtwillen» bescheinigt ${ }^{12}$, daneben freilich ebenfalls dem durch die sowjetische Revolution umgestalteten Russland: «Der eigentliche Feind dieser Revolution ist nicht der Bürger. Kapitalist und Sowjets haben einen gemeinsamen Feind, den Antinaturalisten, Antitechniker, auch Humanisten: Tolstoï» ${ }^{13}$. Sehr richtig erblickt Döblin als weitere Feinde des von ihm umrissenen neuen Lebensgefühls nicht nur den Humanismus auch in der Form der klassischen Bildung, sondern das «Ländliche» ${ }^{14}$; damit ist der Abwehrwille der bedrängten Agrarmentalität gemeint, zu dessen Manifestationen Döblin in undifferenzierter Weise auch den Ku Klux Klan und den Zionismus zählt.

5 An das Ende seines Aufsatzes hat Döblin eine Reflexion über das Verhältnis der «naturalistischen Epoche» zur Ethik gestellt, versehen mit der Überschrift «Hinweis auf Ethisches $»^{15}$. Nachdem er freimütig zugegeben hat, dass die neue Zeit in dieser Hinsicht noch nicht viel zu bieten habe, weil sie erst am Anfang ihrer Entwicklung stehe, versucht er nichtsdestominder zu suggerieren, dass der «Kollektivcharakter» dieser anbrechenden Epoche schon an und für sich ein «ethisches Faktum» darstelle. Offenbar verspricht sich Döblin von der technischen Entwicklung auch die eines neuen Zusammengehörigkeitsgefühls.

Das Vordringen der Sachlichkeit versuchten etwa zur gleichen Zeit, d.h. während der Stabilisierungsperiode, auch die Fachphilosophen als «ethisches Faktum» zu erfassen. Am bekanntesten ist in dieser Hinsicht wohl «Ideologie und Utopie» von Karl Mannheim geblieben (Erscheinungsjahr: 1928). Mannheim ging vom Befund des übergreifenden «Relativismus» und des "völligen Abbaus der Seinstranszendenz» ${ }^{16}$ aus, wobei er als philosophisch geschulter Kopf eine viel eingehendere Analyse vorzulegen vermochte als der in ähnlichen Bahnen denkende Döblin. Die «Sachlichkeit» interpretierte Mannheim vor allem als «Destruktion $»^{17}$ der überkommenen «Ideologien» und «Utopien, aber (und hier lag seiner Meinung nach das Neue), verbunden mit einem keck ausgesprochenen Bekenntnis zur nackten Existenz: «Es gehört eine von unserer Generation kaum mehr vollziehbare Herbheit oder die ahnungslose Naivität einer in diese Welt neu hineingeborene Generation dazu, mit der so gewordenen Wirklichkeit in diesem Sinne in absoluter Deckung zu leben, ohne jede Seinstranszendenz, sei es in der Gestalt der Utopie, sei es in der Gestalt der Ideologie» ${ }^{18}$. Diese Herbheit, der man kraft dieser Beschreibung jeden Anspruch, als sittliche Leistung zu gelten, streitbar machen dürfte, bewertet Mannheim jedoch trotzdem als eine neue Form von Sittlichkeit: «Ist doch die Echtheitskategorie nichts anderes als das 
in das Seelische übertragene Prinzip der Seinskongruenz - das in das Ethische projizierte Prinzip der Sachlichkeit» ${ }^{19}$.

7 Mannheim will es aber - wohlgemerkt - nicht bei der Sachlichkeit bewenden lassen, da er in ihr die Gefahr der «Spannungslosigkeit» erblickt. So kommt es bei ihm zu einer Zurückweisung der Sachlichkeit, weil an ihr «der menschliche Wille zugrunde geh(e)» 20. Mannheim findet sich zwar zur Not noch einigermassen ab mit der «Ideologieenthüllung». Aber das Dahinschwinden der «Utopien» erfüllt ihn mit Bangigkeit. Er sieht den Nihilismus heraufziehen: «Es entstünde die grösste Paradoxie, die denkbar ist, dass nämlich der Mensch der rationalsten Selbstbeherrschung zum Menschen der Triebe wird, dass der Mensch, der nach einer so langen opfervollen und heroischen Entwicklung die höchste Stufe der Bewusstheit erreicht hat - in der Geschichte nicht blindes Schicksal, sondern eigene Schöpfung wird -, mit dem Aufgehen der verschiedenen Gestalten der Utopie den Willen zur Geschichte und damit den Blick in die Geschichte verliert» ${ }^{21}$. Anders und einfacher gesprochen: die Welt der nackten Existenz ist die, wo die Sachzwänge schrankenlos walten. Mannheim selbst befleissigt sich, als Verkünder nicht des Relativismus, sondern des «Relationismus» aufzutreten, indem er, den Boden der Ethik damit immerhin wieder verlassend, die «Gebundenheit des "Wahrheitsbegriffes" an die vorhandenen Wissensarten» betont ${ }^{22}$.

8 Fazit: Mannheim will die Sachlichkeit nur in einem eingeschränkten Sinn gelten lassen, vermag ihr aber letztendlich auch selber nicht zu entrinnen, da die Unterscheidung von «Ideologie» und «Utopie», von Rechtfertigung des Bestehenden und Sprengung des Bestehenden, sich kaum aufrechterhalten lässt: macht doch Mannheim von selbst darauf aufmerksam, dass die verschiedenen noch am Leben gebliebenen Utopien sich gegenseitig mit dem Ideologieverdacht bewerfen ${ }^{23}$.

9 Ganz anders ist die Tonart bei Emil Utitz («Die Überwindung des Expressionismus», 1927). Utitz' Ansichten sind für uns ein verlockendes Thema, weil er zu den wenigen gehört, die sich ausdrücklich zur Neuen Sachlichkeit bekennen. Schon eingangs verkündet er mit entwaffnender Selbstsicherheit: «Ich halte die neue Sachlichkeit oder wie man sonst diese Strömung taufen mag - für den einzigen möglichen Weg: nicht weil andere psychologisch ausgeschlossen sind, sondern weil er allein heute mir geboten scheint, soll nicht die drohende Kulturdämmerung furchtbare Wirklichkeit werden. In dem Kampfe gegen sie bin ich bewusst Partei, allerdings ohne irgendeine Gewähr für den Ausgang des Kampfes zu übernehmen» ${ }^{24}$. Wichtig ist dem Professor Utitz die Zurückdrängung der «Mythes» zugunsten des «Loges», m.a.W. das Bestreben, den politischen Irrationalismus zu bannen. Er beruft sich in diesem Zusammenhang auf den jüngst vollzogenen Sinneswandel von Thomas Mann und zitiert diesbezügliche Stellen aus dessen «Pariser Rechenschaft», um anschliessend hochtrabend aber nichtsdestominder hellsichtig zu proklamieren: «Aus den ärgsten Nöten des Tages, aus wirtschaftlichem und staatlichem Chaos heraus, in das romantisierender Expressionismus wundergläubig steuert, erwacht die Sehnsucht, dass nur wegweisende Klarheit - nicht klügelnde Taktik - führen dürfe. Und über dieser dem Jetzt und Heute zugewandten Rationalität, die gewiss auch von starken und männlichen Gefühlen getragen wird, wölbt sich in ruhiger, ewiger Pracht das Reich des Logos, jene Vernunft die Aristoteles schon preist, und die Schicksal des Abendlandes ist, soll es nicht selbst sich zerstören $»^{25}$.

Immerhin ist sich Utitz der Gefahr durchaus bewusst, dass die neusachliche Haltung unecht, eine «Geste» bleiben könnte, ein «ästhetischer Snobismus» ${ }^{26}$. Daher ist er auch 
bemüht, eine Anzahl von angeblichen Repräsentanten der neusachlichen Gedankenwelt herbeizuzitieren, die uns Späteren freilich als solche gar nicht vorkommen, damals aber einen durchaus respektablen Eindruck machen mussten: Wyneken, Hartmann, Albert Schweizer.

Kohärenter ist allerdings die politische Programmatik, die Utitz der Sachlichkeit beilegt. Er beruft sich auf das amerikanische Gesellschaftsmodell27; seine These lautet: der amerikanische Kapitalismus habe den Gedanken des Dienstes am Ganzen zu versachlichen gewusst, wobei dem Monopolkapitalismus als « grandiose » rationale(r) Sachlichkeit» der Staat als Gegengewicht, und zwar als Träger der "Sittlichkeit» gegenüberstehe. Die Sachlichkeit sei nun zu einer politischen Notwendigkeit im Weltrahmen geworden: sie allein bilde beim einzelnen wie bei den Massen die charakterlichen Voraussetzungen dafür aus, dass die Demokratie überhaupt funktioniere, denn sie erziehe zu Ordnung und Disziplin. Die Zukunft der Menschheit hänge nun einmal davon ab, dass man die politischen Probleme zu versachlichen wisse, um sie unter Kontrolle zu bringen: «Nur eine volle Versachlichung der staatlichen Fragen dient dem Heil, während jede - auch die edelste - Romantik gerade heute Quelle unsagbarer Katastrophen wird ${ }^{28}$. Dass die Sachlichkeit, zumal die amerikanische, sich brutal und gelegentlich verheerend auswirken kann, scheint Utitz nicht zur Kenntnis zu nehmen. Ihm geht es offensichtlich auch darum, die Stabilisierung im Zeichen der «Dollarsonne» (Arthur Rosenberg) mit einer zeitgemässen Ethik zu versorgen. Nicht suspekt ist ihm die Sachlichkeit aber auch aus einem anderen, durchaus ehrbaren Grunde: er fasst sie von vornherein als eine Ethik auf, und zwar als eine überzeitliche, wobei der eingangs bemühte «Logos» für die unabkömmliche Dosis von Transzendenz zu bürgen hat.

12 Utitz hat sich viel Mühe gegeben, um die Neue Sachlichkeit in eine ehrwürdige Tradition einzubetten, in der offenbaren Absicht, den ihr anhaftenden Makel der Modernität zu löschen. Neue Sachlichkeit, das lassen die ersten Kapitel seiner «Uberwindung des Expressionismus » verlauten, ist in ihrer Strenge und Schönheit die Erbin und Wortführerin der deutschen und der sonstigen Klassik. Ernst Bloch schreibt 1935 höhnisch, allerdings ohne den Namen Utitz zu erwähnen: «Eine Reprise von klassizistischer Ruhe und Strenge ging durch die Welt, durch jenes Dasein voll edler Einfalt, stiller Grösse, worin die Kapitalisten leben ${ }^{29}$.

13 Viel weniger geistig anspruchsvoll begegnet uns die neusachliche Ethik bei ihren populären Vertretern. Hier ist die Tendenz auffällig, den Begriff Sachlichkeit als Markenzeichen zu verwenden. Dies scheint mir namentlich der Fall zu sein bei solchen Publizisten Matzke und Wedderkop.

Die Ausführungen von Franz Matzke («Jugend bekennt: So sind wir!», Leipzig, 1930) verdienen unser Interesse schon deshalb, weil dieser sich (wie der geistig höher stehende Professor Utitz) ausdrücklich zur Neuen Sachlichkeit bekennt, und sich dabei auch in löblicher Weise bemüht, bei ihrer Definition um klare Verhältnisse zu sorgen. Die «neue» Sachlichkeit, schreibt er, sei «Sachlichkeit im Lebensstil» und nicht bloss in den Methoden, wo es sie natürlich «immer gegeben hat». Da Lebensstil eine mehr oder minder bewusste Umsetzung eines Lebensgefühls in die Praxis bedeutet, ist es durchaus statthaft, hier einen ethischen Impuls zu diagnostizieren. Das Wort «Lebensstil» scheint mir übrigens nicht sehr glücklich gewählt; angemessener kommt mir die Bezeichnung «Lebenshaltung» vor, die Rudolf Arnheim in seiner Besprechung des Matzke-Buches verwendet ${ }^{30}$. 
15 Matzkes Schilderung der Neuen Sachlichkeit als Lebenshaltung ist aufreizend apodiktisch geschrieben. Es klingt hier etwas durch von der hemmungslosen Rhetorik des «deutschen Jünglings»: Matzkes Ausdrucksweise bringt den inzwischen noch viel sachlicher gewordenen heutigen Leser streckenweise zum Schmunzeln. Es geht ihm offensichtlich darum, jede Form von gefühlsbetonter Kommunikation im menschlichen Verkehr wie im Verhältnis des Subjekts zur Welt als lyrische Empfindsamkeit und verpönte «Beseelung» ostentativ wegzuräumen. Matzke macht dabei von den Worten «Kühle» und «Kälte» ausgiebig Gebrauch. Wieder einmal ist der Hinweis auf das Ethische massgeblich: Matzke labt sich an solchen Begriffen wie «Reinheit» und «Grösse». Es hiesse jedoch, glaube ich, verfehlt denken, in Matzke nur einen Nachplapperer von Döblin und Utitz sehen zu wollen. Manchmal bewegt er sich auch am Rande von geradezu «nationalrevolutionären» Vorstellungen, wie sie namentlich in Jüngers damaligen Essays anzutreffen waren. Kracauer hat in seiner Kritik des MatzkeBuchs $^{31}$ auf eine Stelle aufmerksam gemacht, die uns die Dehnbarkeit der solcherart aufgefassten neusachlichen Ethik und die weiten Möglichkeiten ihrer Vereinnahmung konkret vor Augen führt: «(...) die Unterordnung unter eine Aufgabe, unter ein Ziel (...) bis zur Nichtachtung der eigenen Person. Es ist die Ethik des Sportmanns und des Ozeanfliegers, die Ethik des Soldaten und des Wissenschaftlers: mag mein Leben untergehen, wenn nur das erreicht wird, dem ich diene!» Kracauer interpretierte diese Lebenshaltung als «Flucht» vor der rationalen Auseinandersetzung mit der « gesellschaftlichen Situation und der Notwendigkeit des Wandels $»^{32}$.

Ebenfalls im Namen dieser neuen ethischen Sensibilität verurteilte Hermann von Wedderkop (Herausgeber der neusachlichen Zeitschrift «Der Querschnitt») den ohnehin dahinsiechenden Expressionismus ${ }^{33}$. Auch bei ihm gewinnt die unablässig bemühte «Wirklichkeit » die Qualität einer moralischen Instanz ${ }^{34}$. So gelangen «Kino» und «Zeitung» in den Rang von Mitteln der geistigen Askese, denn sie bewerkstelligen eine «Wirklichkeitsbeschwörung», zu der bei weitem nicht alle geistig Produzierenden berufen sind: denn die meisten unter solchen bleiben befangen in «Bürgerlichem», «Kitsch», "Schlendrian» und verzweifelten Anstrengungen der «Phantasie». Dabei kommt es bei Wedderkop gelegentlich zu lächerlichen Entgleisungen (Wochenschau als «Quintessenz des Zeitgeschehens»). Angestrengte Modernität (Wedderkop brüstet sich mit seinem «Wirklichkeitsfanatismus») ist für unser heutiges Empfinden das Ergebnis: Markenzeichen eben, selbst wenn das dahinterstehende Lebensgefühl Wedderkop nicht schlankerhand abzusprechen ist.

Die Umsetzung dieses ethischen Impulses in einen literarischen lässt sich in fast allen literaturkritischen Textproben verfolgen, die A. Kaes als Dokumente der neuen Sachlichkeit zusammen gestellt hat ${ }^{35}$. So bei Alfred Polgar in seiner Rezension des Hemingway-Erzählungsbands « Men Without Women » (« Das Tagebuch», 14.12.1929). Hier wird aus der literarischen Beurteilung unversehens eine moralische Bewertung: «Hemingways Geschichten sind nackter Realismus, in der Darstellung von einer schon fast romantischen Nüchternheit, ihr geistiges Klima Höhenklima: dünne Luft, Kälte bei intensiver Strahlungswärme. Figuren und Dinge stehen in schneidendem, unbewegtem Licht, ihr Schatten fällt, ohne dass der Dichter ihn gekennzeichnet hätte. Der mischt sich niemals in die Erzählung. Kein Fingerzeig, keine Wertungen, keine Bilder, kein Für und Wider, kein Durchleuchten, keine Pointe (...). Auch an Schilderung wird nur das Existenzminimum gegeben, obschon Hemingway ein grossartiger Schilderer ist ${ }^{36}$. Den gleichen moralisierenden Tonfall finden wir beispielsweise in der Besprechung des 
Romans von Erik Reger «Union der festen Hand» durch Ernst Glaeser («Die literarische Welt», 21.8.1931 $)^{37}$, hier ist gleich eingangs die Rede von einer Literatur neuer Art, «sachlich-brutal, moralischdirekt, ethischschwerfällig», die es nicht abgesehen habe auf stilistische Feinheiten, geniale Formulierungen oder irgendeinen sonstwie bewirkten kultivierten Hochglanz, sondern die schlicht «darstellt und deutet», deren Ethik somit darin besteht, dass sie sich eine rein didaktische Sendung auferlegt. Neben Regers Industrie-Roman lässt Glaeser aus betont ethischen Gründen nur Falladas «Bauern, Bonzen und Bomben», die Lehrstücke von Brecht, die Kriegsromane von Ludwig Renn und Remarque sowie die zeitgenössische angelsächsische Literatur gelten (letztere hatte auch Feuchtwanger zu paradigmatischer Bedeutung erhoben) ${ }^{38}$.

Es wäre natürlich töricht, nun einen Destillationsprozess in Gang zu bringen, um aus den von Glaeser aufgelisteten Texten eine Reihe von moralischen und politischen Verhaltensregeln $\mathrm{zu}$ abstrahieren. Was Glaeser herausstellen will, ist das allen diesen Werken gemeinsame Moment der Erzeugung von Betroffenheit: das Verbindende zwischen ihnen ist der moralische Schock, den sie dem Leser vermitteln und wodurch sie ihn wachrütteln wollen. Diese Betroffenheit ist von eigentümlicher Art, weil ihr ein rationales Moment innewohnt, das eben ein Sondermerkmal der Neuen Sachlichkeit darstellt: dem Leser wird durch die Lektüre dieser Texte zum Bewusstsein geführt, dass er geknechtet ist durch die anonymen Mächte des Kollektivs im technischen Zeitalter, so dass die Lebensaufgabe, die er zu bewältigen hat, die Wiederaneignung seiner Freiheit ist. Dies aber erfordert den Mut zur kritischen Distanz und zur Selbstlosigkeit der analytischen Haltung. Glaeser verwirft auf eine Weise, die tatsächlich etwas Puritanisches an sich hat, das Alibi des «Ewig-Menschlichen» sowie alles, was einen Nachgeschmack von «Belletristik» oder «Unterhaltung» hinterlässt. Er wünscht sich eine schmucklose und engagierte Literatur, die den bürgerlichen Individualismus in seiner bösen, egoistischen Form, wie der Leser ihn in sich verspürt, gründlich wegkuriere. Denn der bürgerliche Individualismus ist längst nicht mehr, so etwa könnten wir Glaesers Gedanken fortentwickeln, das seinerzeit von Kant empfohlene Herausgehen aus der Unmündigkeit, sondern ganz im Gegenteil ein Zurücksinken in diese, wobei die «schöne Literatur» ungeachtet ihrer unbestreitbaren Meisterleistungen eine narkotisierende Rolle spielt. Wie man einer solcherart vorgetragenen aufklärerischen Intention auch gegenüberstehen mag, ihr liegt ein unleugbarer ethischer Ernst zugrunde, sie ist keine Pose. Denn wem sie nicht eignet, wer bloss ihre Diktion imitiert, dem ist die Neue Sachlmichkeit nur eine zynisch anzuwendende Schreibtechnik zur Erzielung von neuartigen Stileffekten, wie sie beispielsweise im Bronnen-Roman «O.S.» zu finden ist ${ }^{39}$.

Vollends in die Sphäre des Ethischen hinaufgehoben sehen wir die Literatur der Neuen Sachlichkeit im Aufsatz «Männliche Literatur von Kurt Pinthus» («Das Tagebuch», 1.6.1929 $)^{40}$. Hier steht neben der Selbstbescheidung, dem Verzicht auf das «Dichten» und die von ihm erzeugte Pathetisierung, der «Antiillusionismus» im Mittelpunkt. Und zwar besteht laut Pinthus die ethische Haltung der neuen Literatur (Roth, Renn, A. Zweig, M.L. Fleisser) gerade in der Tapferkeit, die dazu gehöre, den Bankrott des empfindsamen Humanismus angesichts von Krieg, Inflation, verfehlter oder verhinderter Revolution als «Tatsache» zu akzeptieren und nun von dem Befund der nackten Existenz auszugehen. Literatur sei fürderhin «die unmittelbar gezeigte Krassheit des Zustands und Geschehens». 
21 Diese Haltung paart sich mit einer anderen Form von Askese, nämlich der Abstandnahme vom privatistischen Seelenleiden mit den einhergehenden Selbsterkenntnissen und Ergüssen. In Feuchtwangers Roman «Erfolg» sehen wir bereits 1925 die Reflexionen einer neusachlichen Frau bei der Literaturrezeption abgebildet: «(..) die Romane der Epoche sagten ihr (Johanna Krain) nichts. Sie hatten das Weltbild, die Konventionen der bürgerlichen Gesellschaft zur Voraussetzung und machten ungeheuer viel Gewese, bis einer mit seinen Geschäften Erfolg oder Misserfolg hatte, oder bis einer mit einer schlief $»^{41}$.

In der Endphase der hellsichtigen Ohnmacht bleibt die neusachliche Literatur durch diese ethische Haltung geprägt. Der in der Forschung öfters besprochene grosse Bruch am Anfang der Dreissiger Jahre (Weltwirtschaftskrise, Massenarbeitslosigkeit, Hitlers Aufstieg) bedeutet auf dieser Ebene keinen wirklichen Einschnitt. Die übliche Zweiteilung in eine eher affirmative und eine darauffolgend pessimistische Phase ist nur bedingt stichhaltig. Die Ideen etwa eines Utitz (die übrigens gar nicht so blauäugig optimistisch waren, wie man gemeinhin annimmt) haben auf das Welt- und Menschenbild der neusachlichen Autoren keinen nachhaltigen Einfluss ausgeübt. Desillusioniert war die Neue Sachlichkeit trotz ihres Aufklärungsanliegens bereits vor dem Ende der Stabilisierungsphase. Darüber belehrt uns ein Blick auf die Chronologie: Feuchtwangers «Erfolg» erschien 1925, Ludwig Renns «Krieg» 1928, Remarques «Im Westen nichts Neues» Anfang 1929, Roths «Flucht ohne Ende» und Arnold Zweigs «Streit um den Sergeanten Grischa» 1927. 1927 ist auch das Erscheinungsjahr von Brechts «Hauspostille». 1928 und 1929 (und nicht erst nach dem Ausbruch der Krise) erschienen von Kästner die Gedichtbände «Herz auf Taille» und «Lärm im Spiegel». Die zweite (knappere) Fassung von Horvaths « Sladek » wurde im Oktober 1929 uraufgeführt.

Der Neuen Sachlichkeit wurde in der Endphase der Republik ihr nihilistisches Gehabe vorgeworfen: Nihilismus, zur Schau getragen, als Markenzeichen auf Konsumartikeln. Anvisiert wurde die Kaltschnäuzigkeit des neusachlichen Diskurses, und zwar vorwiegend in der Form, die er bei Kästner und Irmgard Keun annahm. Dabei wurde aber der despektierliche Tonfall ungebührend mit einem Mangel an innerer Teilnahme gleichgesetzt. War nun in der Tat der ethische Impuls abgelöst durch eine schnöde, leicht zu vermarktende moralische Indifferenz?

Doch will das Fazit der entzauberten Existenz und der durch Industrialismus und Bürokratismus bewirkten Atomisierung der Gesellschaft noch lange nicht heissen, dass etwa dem Lyriker Kästner kein besseres Menschenbild vorschwebt oder dass er sich aller sittlicher Wertvorstellungen entledigt hat. Das in ethischer Hinsicht immerhin Bedenkliche ist im Grunde Kästners Mischung von «Süffisanz und Fatalismus», dit Walter Benjamin in seinem Artikel «Linke Melancholie» anprangerte ${ }^{42}$. Benjamins Kritik trifft - sehen wir einmal von ihrer politischen Intention ab - den ethischen Kern der Neuen Sachlichkeit, den Anspruch auf Echtheit. Insofern ist sie auch durchaus anwendbar auf Werke anderer neusachlicher Autoren in derselben Zeit, auf Horvaths «Italienische Nacht», Irmgard Keuns «Gilgi» und ihr «Kunstseidenes Mädchen» und Falladas «Bauern, Bonzen und Bomben». Alle diese Werke, die den literarischen Ruhm der Neuen Sachlichkeit für die Nachwelt erst wirklich begründeten, kennzeichnet der politische Überdruss an der Unzulänglichkeit der Weimarer Führungseliten und «Bonzen», die Abkehr vom politischen Kampf, der sich zunehmend polarisiert; die Regignation geht dabei Hand in Hand mit der Zurschaustellung der eigenen Fähigkeit, 
eine hoffnungslos verfahrene, dem Chaos entgegentreibende politische und wirtschaftliche Lage in spannende Lektüre oder witzigen Unterhaltungsstoff $\mathrm{zu}$ verwandeln. Bei Kästners Gedichten wird, folgt man dem Gedankengang Benjamins, der ethische Anspruch zunichte gemacht auch durch die Beschaffenheit des speziellen Lesepublikums, das Kästner ansprechen will: Angehörige der «Zwischenschicht Agenten, Journalisten, Personalchefs»; lauter Wendriners also, möchte man meinen, hätte Benjamin nicht auch ausdrücklich den Kurt Tucholsky im selben Artikel unter Beschuss genommen.

Weniger anspruchsvoll als Benjamins Rezension des Kästner Gedichtbands «Ein Mann gibt Anskunft» liest sich Bernhard von Brentanos Verriss des Keun-Romans «Gilgi» in der «Linkskurve»" ${ }^{43}$, der ebenfalls die Diagnose des Ethikdefizits stellen zu dürfen meint. Der Autorin wird "Indifferenz " gegen die Leiden der Masse vorgeworfen, da die neusachliche Heldin sich sowieso allein durchbringe und dabei am Ende auch über alles hinwegsetze, was ihr «lästig» sei, einschliesslich besagten Leidens.

In der Tat sind die ethischen Ansprüche, die Gilgi und Olga, die beiden neusachlichen Frauen, an sich und an die anderen stellen, die Werte des ungeschminkten bürgerlichen Individualismus aus einer Zeit, wo es in ökonomischer Hinsicht aufwärts ging: Selbständigkeits- und Durchsetzungswille, Arbeitsamkeit und Rührigkeit, Selbstdisziplin, Skepsis gegenüber romantisierenden Klischees und idealistischen Phrasen. Es kann freilich nicht davon die Rede sein, dass Gilgi bzw. die Autorin Irmgard Keun gleichgütlig bleiden gegen das Elend, das sie umgibt. Aber der Autonomienanspruch bleibt massgeblich, selbst wenn Gilgi sich konfrontiert sieht mit der verzweifelten Lage der Arbeitslosen: «Elend und Armut, das ist vielleicht nicht das Schlimmste. Das Schlimmste ist, dass man den Menschen hier jedes Verantwortungsgefühl genommen hat. Das Schlimmste ist, dass manche sind, die sich beinahe behaglich fühlen in dem «Ich kann nichts dafür» - betten sich in den Begriff ausschliesslich fremder Schuld am eigenen Elend wie in einem Sarg. Lassen sich das gute, gute Wissen um eigene Trägheit und Fehlerhaftigkeit morden, lassen Lebenswillen und Kraftwunsch langsam in sich sterben (...)» $»^{44}$. Es steckt in dieser Gesinnung schon ein tüchtiges Stück säkularisierten «Puritanertums». Die Kennzeichnung der Neuen Sachlichkeit durch Ernst Bloch, die wir eingangs zitierten, ist (vorausgesetzt freilich, dass man sie aus ihrem bizarren Kontext herauslöst) gar nicht so abwegig, sie scheint jedenfalls vorzüglich zu passen auf die Gilgi-Figur: «ein puritanischer Zug (...) mitten im kessesten Schein». Bei aller zur Schau getragenen sexuellen Freizügigkeit hat das Mädchen Gilgi fast alle Eigenschaften, die der Freiburger Professor G. von Schulze Gaewernitz beim englischen Puritaner feststellte: «Selbstbeherrschung und Selbstvertrauen, Unabhängigkeit von der Meinung und der Hilfe anderer, Unabhängigkeit aber auch von den eigenen Affekten, Planmässigkeit der Lebensführung, Misstrauen gegen alles rein Gefühlsmässige und Instinktive bezeichnen diesen stahlharten Menschen, welcher im 16. Jahrhundert auf die geschichtliche Bühne hervortritt»" ${ }^{45}$. Gilgi vertritt die frühe bürgerliche Ideologie der «self-reliance» in Reinkultur: Autonomie des Gewissens plus die Eigenschaften des unabhängigen Unternehmers. Jeder Art von Denken mit Ausrichtung auf das bergende Kollktiv ist dies ein Schlag ins Gesicht. Eine ähnliche neusachliche Härte legte die Revolutionärin Eva Berg schon 1927 an den Tag in Tollers «Hoppla, wir leben!» (vgl. 2. Akt, 1. Szene).

In Kästners Fabian ist das Ethische schon im Untertitel angekündigt: «Die Geschichte eines Moralisten». Fabian ist, an Gilgi gemessen, ein weicher Mensch. Die Grundhaltung 
des «Moralisten» ist, so Lethen, die witzige Passivität, betrieben als permente Selbstaufwertung ${ }^{46}$. Die linksgerichtete Kritik hat damit ein leichtes Spiel. Der ethische Anspruch, soweit er vorhanden ist, scheint sich bei Fabian gleichsam von selbst aufzuheben. Darf man also Egon Schwarz beipflichten, wenn er trotzdem Kästner den Verdienst attestiert, er habe «dem Lebensgefühl des Weimarer Humanisten unverwechselbaren und unvergesslichen Ausdruck» gegeben? ${ }^{47}$

Man könnte im Falle des «Fabian» durchaus geltend machen, dass Kästner sich zu den gängigen Grundsätzen bürgerlichen Zusammenlebens bekennt. Bei ihm, darauf hat R.W. Leonhardt hingewiesen, kann sogar «keine Rede (...) von einer Moral der Emanzipation (...) sein $»^{48}$. Schmerzhaft erlebt Fabian die Auflösung der moralischen Werte, die als Regulative für jede echte Demokratie unentbehrlich sind. Es ist aus mit der Festigkeit der Ehe, der Reinheit der Liebe, dem ehrlichen kollegialen Verhalten, der Würdigung des Verdienstes, der wahrheitsgemässen Aufklärung des Publikums durch eine freie, das allgemeine Interesse stets im Auge behaltende Presse. Man kann es immerhin verstehen, dass die provozierende und pikante Art, wie Kästner diesen Befund ausbreitet, auf strenge Gemüter eher kulinarisch wirkt. Die Grundfrage aber, die der «Fabian» auch noch an uns stellt, die wir 50 Jahre später leben, lautet: Ist eine ethische Haltung überhaupt noch denkbar in einer Welt der Sachzwänge und der Verlogenheit, wo das Menschenschicksal in ethischer Hinsicht zunehmend sinnlos wird, deren Gang Kants Postulate der praktischen Vernunft auf Schritt und Tritt Lügen straft?

29 Als die westliche Welt nach 1945 dank dem Marschall-Plan und neuer Lebenslügen wieder ins Lot kam, setzte mann Labudes neusachliches Ideal des aufgeklärten Wohlfahrtsstaats in die Praxis um, solange zumindest, wie die Konjunktur auf dem Arbeitsmarkt es erlaubte. Es entstand gleichzeitig von neuem der Himmel des Eigentums, in den in Horvaths «Italienischer Nacht» die Leni ihren Karl hinanzieht ${ }^{49}$.

Einen unangenehmen Eindruck machte die Neue Sachlichkeit eigentlich eben eher dadurch auf die Gemüter, dass sie am Ethischen klebte und sich hartnäckig der parteilichen Bindung versagte. In dieser Hinsicht ist Döblins Schrift «Wissen und Verändern» eines der grossen Zeugnisse neusachlicher Ethik ${ }^{50}$. Durch diese Weigerung, sich direkt am politischen Kampf zu beteiligen, zog sich die Neue Sachlichkeit den Vorwurf zu, dass sie von ihrem Aufklärungsanliegen abrückte. Es war nun ein Leichtes für die linksengagierte Kritik (die Kritik von rechts braucht uns hier nicht zu bekümmern, denn sie hatte die Neue Sachlichkeit schon längst als Nihilismus abgeurteilt), die neusachlichen Autoren an ihren eigenen Ansprüchen zu messen und ihre Einstellungen als Narzissmus anmassender, feixender Intellektueller oder als zynisches Vermarktungsunternehmen von Katastrophenstimmungen $\mathrm{zu}$ disqualifizieren.

Die Angriffsflächen waren freilich im Projekt der Neuen Sachlichkeit vorprogrammiert. Denn man wollte ja einerseits für die Massen schreiben, die politisch-gesellschaftliche Veränderung bewirken helfen, doch gleichzeitig wollte man sich die Autonomie bewahren, die den geistig Schaffenden des bürgerlichen Europa vom pflichttreuen sowjetischen Texte-Schreiber unterschied ${ }^{51}$. Die Neue Sachlichkeit hat bis zum Ende unter dieser Spannung gelitten; oder vielmehr, es wurde von ihr Unmögliches verlangt, nämlich dass sie, die sich die Aufgabe gestellt hatte, den Zusammenstoss des Einzelmenschen mit dem Kollektiv aufklärend darzustellen, sofort auch 
Handlungsanleitungen gebe, was für die Literatur nun nichts anderes bedeuten kann, als sich unter parteipolitischen Rechtfertigungszwang zu stellen.

Als Fortsetzer des Aufklärungshumanismus hielten sich aber die neusachlichen Autoren beharrlich an eine Gesinnungsethik und vermieden es, mit der Verantwortungsethik zu paktieren. So wurde wieder einmal der Nachweis erbracht, dass die Literatur, sofern sie diesen Namen verdient, unbestechlich bleibt.

\section{NOTES}

1. - Siehe Leo Lania, «Reportage als soziale Funktion», in Die literarische Welt, 25.6.1926, abgedruckt bei Kaes, «Manifeste und Dokumente der deutschen Literatur 1918-1933», Metzler, Stuttgart, 1983, S. 323: «Kisch fühlt sich nur als Knecht des darzustellenden Objekts».

2. - Horst Denkler, «Sache und Stil. Die Theorie der "Neuen Sachlichkeit" und ihre Auswirkungen auf Kunst und Dichtung», in Wirkendes Wort, 18, 1960, S. 176.

3. - Ernst Bloch, «Erbschaft dieser Zeit», erweiterte Ausg., Suhrkamp Verlag, Frankfurt-am-Main, 1962, S. 217.

4. - Siegfried Kracauer, «Katholizismus und Relativismus», 1921, in: «Das Ornament der Masse», Suhrkamp taschenbuch 371, 2. 194.

5. - Kracauer, «Die Wartenden», a.a.O., S. 106-119.

6. - Alfred Döblin, «Futuristische Worttechnik» und «An Romanautoren und ihre Kritiker. Berliner Programme», in: «Aufsätze zur Literatur», Ausgewählte Werke in Einzelbänden, hrsg. v. Walter Muschg, Walter Verlag, Ölten und Freiburg-im-Breisgau, 1953.

7. - In: «Der Geist des naturalistischen Zeitalters», a.a.O., S. 66.

8. - In: «An Romanautoren und ihre Kritiker», a.a.O., S. 18.

9. - «Der Geist des naturalistischen Zeitalters», a.a.O., S. 63, 65.

10. - a.a.0., S. 66.

11. - a.a.O., S. 67-68.

12. - a.a.O., S. 74 .

13. - a.a.O., S. 76.

14. - a.a.O., S. 81.

15. - a.a.O., S. 83.

16. - Karl Mannheim, «Ideologie und Utopie», l.u.2. Auflage 1929; 7. Aufl. bei Vittorio Klostermann, Frankfurt-am-Main, 1985, S. 219.

17. - a.a.O., S. 220.

18. - ebda.

19. - ebda

20. - a.a.O., S. 224-225.

21. - a.a.O., S. 225.

22. - a.a.O., S. 250.

23. - a.a.O., S. 213-216.

24. - Emil Utitz, «Die Überwindung des Expressionismus», Verlag von Ferdinand Enke, Stuttgart, 1927, S. 9.

25. - a.a.O., S. 45.

26. - a.a.O., S. 93-94. 
27. - a.a.O., S. $176 f$.

28. - a.a.O., S. 180 .

29. - Bloch, a.a.O., S. 217.

30. - R. Arnheim, «Die Gefühle der Jugend», in: «Die Weltbühne», 27.1.1931, S. 137.

31. - S. Kracauer, «Neue Jugend?», in: «Neue Rundschau», Januar, 1931, zitiert bei Kaas, a.a.O., S. 186-187.

32. - a.a.O., S. 187.

33. - «Expressionismus und Wirklichkeit», Oktober 1921, «Wandlungen des Geschmacks», 1926; bei Kaes, a.a.O., S. 159-161, 252-254.

34. - In: «Wandlungen des Geschmacks».

35. - Siehe Kaes, a.a.O., S. 319-482: «Die Funktionskrise der reinen Kunst: Literarischer Formenwandel und Publikumsbezug».

36. - Bei Kaes, a.a.O., S. 391.

37. - a.a.O., S. 193-195.

38. - L. Feuchtwanger, «Ein Buch für meine Freunde» («Centum Opuscula»), Frankfurt-am-Main, 1984; Aufsatz "Von den Wirkungen und Besonderheiten des angelsächsischen Schriftstellers», 1928, S. 417-421.

39. - Vgl. Kurt Tucholskys Rezension des Romans: «Ein besserer Herr», Weltbühne, 6, 1929. Auch abgedruckt in K. Tucholsky, «Literaturkritik», hrsg. von F.J. Raddatz, rowohlt-Taschenbuch 5548, S. 223-230.

40. - Bei Kaes, a.a.O., S. 328-333.

41. - L. Feuchtwanger, «Erfolg. Drei Jahre Geschichte einer Provinz», Fischer Taschenbücherei 1680 , S. 385

42. - 1931. In: W. Benjamin, Schriften III, Suhrkamp, Frankfurt-am-Main, 1972.

43. - «Keine von uns. Ein Wort an die Leser des "Vorwärts"», in: Linkskurve, 4.10.1932, abgedruckt bei Kaes, S. 357-258.

44. - «Gilgi - eine von uns», 1931. Zitiert wird die 3. Neuauflage, Bastei-Lübbe Taschenbuch, 11160, S. 155.

45. - G. von Schulze-Gaewernitz, o. Prof. der Nationalökonomie an der Universität Freiburg-in-B., «Britischer Imperialismus und englischer Freihandel zu Beginn des zwanzigsten Jahrhunderts», Dunckler und Humbolt, Leipzig, 1906, S. 29.

46. - H. Lethen, «Neue Sachlichkeit», 2. Aufl., Metzler, Stuttgart, 1975, S. 142-155: «Kästners Fabian oder die Karikatur freischwebender Intelligenz».

47. - Egon Schwarz, «Fabians Schneckengang im Kreise», in: «Zeitkritische Romane des XX. Jahrhunderts», Reclam, Stuttgart, 1975, S. 124-145.

48. - R.W. Leonhardt, «Fabian alias Kästner», in: «Die Zeit», 23.5.1980.

49. - «Italienische Nacht», Suhrkamp Verlag, 1974, S. 50 (Sechstes Bild).

50. - Siehe die (sehr kritische) Analyse von W. Köpke in «Weimars Ende», Suhrkamp taschenbuch 2019, 1982, S. 318-329.

51. - Vgl. die beiden Artikel von S. Kracauer, «Über den Schriftsteller» («Neue Rundschau», Juni 1931, und «Instruktionsstunde in Literatur. Zu einem Vortrag des Russen Tretjakow», «Frankfurter Zeitung», 26.4.1931, auszugsweise abgedruckt bei Kaes, a.a.O., S. 190-193). 


\section{RÉSUMÉS}

Sachlichkeit und Ethik scheinen unvereinbare Begriffe zu sein, es drängt sich jedoch bei der Lektüre der neusachlichen Literatur und Philosophie die Einsicht auf, daß es der Neuen Schlichkeit in ihren bedeutendsten Hervorbringungen um etwas anderes und Höheres ging als um die Verbreitung von technokratischen Standpunkten, die Verulkung von Romantik und Expressionismus oder die Ästhetisierung von Industrie- und Arbeitswelt. Wirklichkeit als ethische Instanz, das bedeutet Überwindung des heuchelnden Idealismus der Vorkriegszeit sowie Abweisung des expressionistischen Humanitätsschwulstes. Angestrebt wird ein unsentimentaler Humanismus, der jeder Narkotisierung von rechts- und linksradikaler Seite entgegenwirken soll.

L'attitude objectiviste et le vouloir éthique - ces deux notions ne semblent guère s'accorder. Mais lorsqu'on lit les textes littéraires et philosophiques de la Neue Sachlichkeit, on s'aperçoit forcément que la "Nouvelle Objectivité », dans ses œuvres majeures, recherche autre chose et vise plus haut que la diffusion de vues technocratiques, la satire bouffonne du romantisme et de l'expressionnisme ou la vision unilatéralement esthétique du monde industriel et du monde du travail. La réalité érigée en instance morale, cela signifie qu'on veut dépasser l'idéalisme hypocrite de l'avant-guerre, et que l'on refuse de même l'emphase humanitaire de l'expressionnisme. Ce qui est recherché, c'est un humanisme dépourvu de sentimentalité, qui puisse s'opposer aux drogues idéologiques de l'extrême-droite et de l'extrême-gauche.

\section{AUTEUR}

\section{PIERRE VAYDAT}

Universität Charles de Gaulle - Lille III 